\title{
Partial Tmem106b reduction does not correct abnormalities due to progranulin haploinsufficiency
}

\author{
Andrew E. Arrant ${ }^{1}$, Alexandra M. Nicholson ${ }^{2}$, Xiaolai Zhou ${ }^{2}$, Rosa Rademakers ${ }^{2^{*}}$ and Erik D. Roberson ${ }^{1 *}$ (D)
}

\begin{abstract}
Background: Loss of function mutations in progranulin (GRN) are a major cause of frontotemporal dementia (FTD). Progranulin is a secreted glycoprotein that localizes to lysosomes and is critical for proper lysosomal function. Heterozygous GRN mutation carriers develop FTD with TDP-43 pathology and exhibit signs of lysosomal dysfunction in the brain, with increased levels of lysosomal proteins and lipofuscin accumulation. Homozygous GRN mutation carriers develop neuronal ceroid lipofuscinosis (NCL), an earlier-onset lysosomal storage disorder caused by severe lysosomal dysfunction. Multiple genome-wide association studies have shown that risk of FTD in GRN mutation carriers is modified by polymorphisms in TMEM106B, which encodes a lysosomal membrane protein. Risk alleles of TMEM106B may increase TMEM106B levels through a variety of mechanisms. Brains from FTD patients with GRN mutations exhibit increased TMEM106B expression, and protective TMEM106B polymorphisms are associated with decreased TMEM106B expression. Together, these data raise the possibility that reduction of TMEM106B levels may protect against the pathogenic effects of progranulin haploinsufficiency.
\end{abstract}

Methods: We crossed Tmem $106 b^{+/-}$mice with $\mathrm{Grn}^{+/-}$mice, which model the progranulin haploinsufficiency of GRN mutation carriers and develop age-dependent social deficits and lysosomal abnormalities in the brain. We tested whether partial Tmem106b reduction could normalize the social deficits and lysosomal abnormalities of $\mathrm{Grn}^{+/-}$mice.

Results: Partial reduction of Tmem106b levels did not correct the social deficits of $\mathrm{Grn}^{+/-}$mice. Tmem106b reduction also failed to normalize most lysosomal abnormalities of $\mathrm{Grn}^{+/-}$mice, except for $\beta$-glucuronidase activity, which was suppressed by Tmem106b reduction and increased by progranulin insufficiency.

Conclusions: These data do not support the hypothesis that Tmem106b reduction protects against the pathogenic effects of progranulin haploinsufficiency, but do show that Tmem106b reduction normalizes some lysosomal phenotypes in $\mathrm{Grn}^{+-}$mice.

Keywords: Progranulin, TMEM106B, Frontotemporal dementia, Lysosome

\section{Background}

Progranulin (GRN) is a secreted glycoprotein that acts as a growth factor for many cell types, modulates inflammation, and is critical for proper lysosomal function [1-5]. Lossof-function GRN mutations are one of the most common genetic causes of frontotemporal dementia (FTD), causing

\footnotetext{
* Correspondence: rademakers.rosa@mayo.edu; eroberson@uabmc.edu ${ }^{2}$ Department of Neuroscience, Mayo Clinic Jacksonville, 4500 San Pablo Road, Jacksonville, FL, USA

${ }^{1}$ Center for Neurodegeneration and Experimental Therapeutics, Alzheimer's Disease Center, Evelyn F. McKnight Brain Institute, Departments of Neurology and Neurobiology, University of Alabama at Birmingham, 1825 University Blvd., SHEL, Birmingham, AL 1110, USA
}

as much as $5-10 \%$ of all FTD cases and $20 \%$ of familial FTD cases [6-8]. GRN mutations are an autosomal dominant cause of FTD, and most known disease-causing mutations lead to haploinsufficiency of progranulin, with GRN carriers typically having less than $50 \%$ of normal circulating progranulin levels [6-9]. Progranulin haploinsufficiency is therefore thought to cause FTD in GRN mutation carriers. GRN mutations were thought to be highly penetrant until the identification of TMEM106B variants as an important modifier of disease risk in GRN carriers [10-13].

TMEM106B was first identified as a genetic risk factor for FTD with TDP-43 pathology (FTD-TDP) when a

(C) The Author(s). 2018 Open Access This article is distributed under the terms of the Creative Commons Attribution 4.0 International License (http://creativecommons.org/licenses/by/4.0/), which permits unrestricted use, distribution, and 
genome-wide association study found three single nucleotide polymorphisms (SNPs) associated with FTD-TDP risk in the region of chromosome 7 that contains TMEM106B [10]. Subsequent analyses identified these SNPs to be more strongly associated with FTD risk in individuals with GRN mutations [10], an observation that was replicated in several additional studies [11-13]. Since this initial discovery, TMEM106B SNPs were also found to modify disease risk in patients carrying FTD-causing C9ORF72 repeat expansions; however, the strongest association of TMEM106B SNPs with FTD risk has been repeatedly found in GRN mutation carriers [10-16]. Thus, many genetic studies show a clear relationship of TMEM106B and GRN in FTD pathogenesis.

The mechanism by which TMEM106B SNPs may modify FTD risk in GRN carriers is unclear, though current data suggest that protective SNPs may reduce TMEM106B mRNA expression or protein levels. The protective allele of the SNP with the strongest FTD association, rs1990622, may be associated with lower TMEM106B expression $[10,17]$, though this has not been replicated by some other studies $[11,13,18]$. Evaluation of the TMEM106B SNP rs1990620, which is in complete linkage disequilibrium with rs1990622, revealed that the protective allele of rs1990620 facilitates binding of the transcriptional repressor CCCTC binding factor to TMEM106B, resulting in lower TMEM106B expression [17]. Independent analysis of a coding SNP (rs3173615 or p.T185S) in complete linkage disequilibrium with rs1990622 revealed that the protective (S) allele results in faster degradation of TMEM106B protein [19]. As a result, protein levels of the risk (T) TMEM106B allele were approximately twice as high as those of the protective (S) allele when expressed in a cell line, despite similar TMEM106B mRNA levels [19]. Perhaps consistent with a protective role of lower TMEM106B levels, increased levels of TMEM106B mRNA and protein have been observed in brains from FTD-TDP patients, both with and without GRN mutations [10, 20, 21]. Together, these studies form the basis of the hypothesis that reduction of TMEM106B levels may protect against the pathogenic effects of progranulin haploinsufficiency.

Reduction of TMEM106B levels might protect GRN mutation carriers against FTD by improving lysosomal function. Lysosomal dysfunction may be key to FTD-GRN pathogenesis, as progranulin is critical for proper lysosomal function. Individuals homozygous for loss of function GRN mutations that cause nearly complete progranulin deficiency develop the lysosomal storage disorder, neuronal ceroid lipofuscinosis [22, 23]. FTD patients with GRN mutations also exhibit signs of lysosomal dysfunction, such as increased levels of lysosomal proteins and lipofuscin accumulation in the brain and retina [21, 24, 25]. TMEM106B is a lysosomal membrane protein, and TMEM106B overexpression in cell culture has detrimental effects on lysosomal function, causing lysosomal enlargement, impaired acidification, reduced motility, and clustering of lysosomes in the cell body of neurons [17, 20, 26-28]. TMEM106B overexpression may increase progranulin levels, though this is likely due to lysosomal dysfunction, as lysosome-disrupting compounds also increase progranulin levels [20, 27, 29]. Although TMEM106B knockdown has been shown to affect dendritic lysosomal trafficking, lowering TMEM106B levels reduces lysosomal size and number $[28,30]$. Taken together, these cell biology studies of TMEM106B indicate that reduction of TMEM106B could ameliorate the lysosomal dysfunction of GRN mutation carriers.

The available genetic and cell biological data indicate that reducing TMEM106B levels may have beneficial effects in GRN mutation carriers. In this study, we tested this hypothesis using $\mathrm{Grn}^{+/-}$mice. $\mathrm{Grn}^{+/-}$mice model the progranulin haploinsufficiency that causes FTD in GRN mutation carriers, and develop age-dependent social behavior deficits and lysosomal dysfunction in the brain [31-33]. Recent studies have tested the effects of Tmem106b overexpression or deletion in $\mathrm{Grn}^{-/}$mice, which develop severe lysosomal dysfunction and lipofuscinosis that may model the pathology of NCL in homozygous GRN mutation carriers. Consistent with prior cell biology studies, overexpression of human TMEM106B worsened the lipofuscinosis and lysosomal abnormalities of $\mathrm{Grn}^{-/-}$mice [34]. In contrast, knockout of Tmem106b in mice suppresses expression of many lysosomal enzymes, which opposes the increases in lysosomal enzyme expression caused by progranulin deficiency [35]. As a result, crossing $\mathrm{Grn}^{-/-}$mice with Tmem106b ${ }^{-/-}$mice normalized the activity of some lysosomal enzymes in $\mathrm{Grn}^{-1-}$ mouse brain, as well as open field and elevated plus maze behavior, though it failed to normalize lipofuscinosis and gliosis [35]. However, TMEM106B polymorphisms only partially reduce TMEM106B expression [19], so in this study, we crossed Tmem $106 b^{+/-}$mice with $\mathrm{Grn}^{+/-}$mice to test whether partial reduction of Tmem106b would correct the behavioral deficits and lysosomal dysfunction of $\mathrm{Grn}^{+/-}$ mice, which model the progranulin haploinsufficiency that causes FTD-GRN [31-33].

\section{Methods}

Generation of $\mathrm{Grn}^{+/-}$:Tmem $106 b^{+/-}$mice

Tmem $106 b^{+/-}$mice were generated with a previously described gene trap vector developed by the Wellcome Trust Sanger Institute [35] and bred onto a C57BL/6 N background. $\mathrm{Grn}^{+/-}:$Tmem $106 b^{+/-}$mice were generated by crossing $T m e m 106 b^{+/-}$mice with $\mathrm{Grn}^{+/-}$mice on a C57BL/6 J background that were generated as previously described $[31,36]$. The F1 generation was used for this study. This pairing resulted in four possible

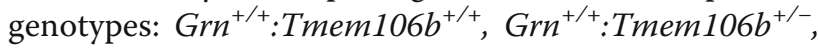




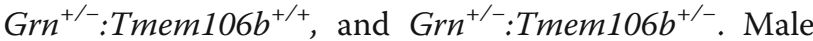
and female littermates from these pairings were used for all studies, with $\mathrm{Grn}^{+/+}:$Tmem $106 b^{+/+}$mice serving as the control group. Mice were housed in the University of Alabama at Birmingham (UAB) mouse housing facility accredited by the Association for Advancement and Accreditation of Laboratory Animal Care International. The mice were maintained on a 12:12 h light/dark schedule, with the lights on at 6:00 AM and off at 6:00 PM. All experiments were conducted during the light phase under ambient room lighting. Mice were given free access to food (Harlan, \#7917) and water throughout the experiment. All experiments were approved by the UAB Institutional Animal Care and Use Committee.

\section{Tube test for social dominance}

The tube test for social dominance was conducted on both male and female mice as previously described [32], with mice of the same sex but different genotype placed in opposite ends of a $30.5 \mathrm{~cm}$-long clear plastic tube. The mice were released, and the first mouse to have two feet out of the tube was considered to have lost the match. Mice were tested at age 12-14 months, an age at which $\mathrm{Grn}^{+/-}$mice exhibit a stable low-dominance phenotype [32]. To confirm the presence of the expected $\mathrm{Grn}^{+/-}$phenotype, we paired $\mathrm{Grn}^{+/+}: \mathrm{Tmem}_{106 b^{+/+}}$mice with $\mathrm{Grn}^{+/-}:$Tmem $106 b^{+/+}$mice. To test the effect of Tmem106b reduction on the $\mathrm{Grn}^{+/-}$phenotype, we paired $\mathrm{Grn}^{+/+}:{\text {Tmem } 106 b^{+/+}}$mice with $\mathrm{Grn}^{+/-}:$Tmem106b $^{+/-}$ mice. In both tests, each mouse was paired against three other mice of the opposing genotype over three rounds of testing, as previously described [32]. Matches from each test $\left(\mathrm{Grn}^{+/+}:\right.$Tmem106b $^{+/+}$vs. Grn ${ }^{+/-}:$Tmem106b $^{+/+}$ or $\mathrm{Grn}^{+/-}: \mathrm{Tmem}_{\left.106 b^{+/-}\right)}$were interspersed to avoid any confounding effects of test order. For example a $\mathrm{Grn}^{+/+}: \mathrm{Tmem} 106 \mathrm{~b}^{+/+}$mouse would face a $\mathrm{Grn}^{+/-}$: $\mathrm{Tmem} 106 b^{+/+}$mouse in round 1 of testing, then a $\mathrm{Grn}^{+/-}$: Tmem $106 b^{+/-}$mouse in round 2, with the genotype of the opponent alternating over a total of six rounds of testing. We did not observe an effect of testing round on the dominance phenotypes, so the repeated testing did not measurably affect the behavioral performance of the mice, as we have previously observed [32]. On a separate day, we paired $\mathrm{Grn}^{+/-}: \mathrm{Tmem}_{106 b^{+/+}}$mice against $\mathrm{Grn}^{+/-}$: Tmem $106 b^{+/-}$mice, and $\mathrm{Grn}^{+/+}:$Tmem $106 b^{+/+}$mice against $\mathrm{Grn}^{+/+}:$Tmem $106 b^{+/-}$mice to assess the effect of Tmem106b reduction on social dominance within $\mathrm{Grn}^{+/-}$mice. This test was also conducted over three rounds of testing.

\section{Western blot}

The following antibodies were used for western blot: LAMP-1 (1:250, Developmental Studies Hybridoma Bank \#1D4B), Tmem106b (1:500, Bethyl Laboratories,
\#A303-439A), progranulin (1:500, rabbit anti-progranulin polyclonal antibody [37]), HexA (1:500, Abcam, \#189685), GCase (1:500, Santa Cruz, \#sc-166,407), GAPDH (1:5000, MilliporeSigma \#MAB374). All primary antibody incubations were conducted overnight at $4{ }^{\circ} \mathrm{C}$.

Frontal cortex samples were homogenized in lysis buffer (50 mM Tris, $150 \mathrm{mM} \mathrm{NaCl}, 5 \mathrm{mM}$ EDTA, 1\% Triton $\mathrm{X}-100,0.1 \%$ sodium deoxycholate) and centrifuged at $5000 \times \mathrm{g}$ for $10 \mathrm{~min}$ at $4{ }^{\circ} \mathrm{C}$. Protein concentration of the lysates was assessed by Bradford assay (Coomassie Plus, Thermo Scientific). For western blot, samples were diluted with 4X LDS sample buffer (ThermoFisher Scientific) and 10X Bolt sample reducing agent (ThermoFisher Scientific) and heated for $10 \mathrm{~min}$ at $70{ }^{\circ} \mathrm{C} .20 \mu \mathrm{g}$ of protein was loaded per lane on $4-12 \%$ bis-tris gels. After electrophoresis, proteins were transferred to Immobilon-FL PVDF membranes (MilliporeSigma) and blocked with 50\% Odyssey blocking buffer (LI-COR Biotechnologies) prior to overnight incubation with primary antibody. Antibody labeling was detected using an IR-dye-conjugated species-matched secondary antibody (LI-COR Biotechnologies).

Western blot of Tmem106b in brain tissue proved to be incompatible with bis-tris polyacrylamide gels. For these blots, $20-30 \mu \mathrm{g}$ of protein was loaded onto $12 \%$ TGX gels (Biorad) and transferred onto Immobilon-FL PVDF membranes (MilliporeSigma) in Dunn carbonate buffer [38]. The membranes were blocked in 5\% milk prior to probing with antibodies as described above. All blots were scanned on an Odyssey scanner (LI-COR Biotechnologies) and quantitated with Image Studio Lite software (LI-COR Biotechnologies).

\section{Enzyme activity assays}

Fluorogenic assays were used to determine the enzymatic activity of $\beta$-hexosaminidase A (HexA), $\beta$-glucocerebrosidase (GCase), and $\beta$-glucuronidase (Gusb) in frontal cortical lysates. Frontal cortex samples were prepared without protease inhibitors as described for western blot. HexA and Gusb activity were determined by incubating frontal cortical lysates $(5 \mu \mathrm{g}$ protein per well) with $2 \mathrm{mM}$ fluoregenic substrates for HexA (4-methylumbelliferyl-2acetamido-2-deoxy-6-sulfate- $\beta$-D-glucopyranoside, Research Products International) or Gusb (4-methylumbelliferyl- $\beta$-Dglucuronide hydrate, MilliporeSigma) in $10 \mathrm{mM}$ sodium citrate buffer, $\mathrm{pH} 4.2$ [39]. The reactions proceeded for $1 \mathrm{~h}$ at $37{ }^{\circ} \mathrm{C}$ and stopped by addition of $0.2 \mathrm{M}$ glycine, $0.2 \mathrm{M}$ sodium carbonate. GCase activity was determined by incubating frontal cortical lysates $(10 \mu \mathrm{g}$ protein per well) with $1 \mathrm{mM}$ fluorogenic GCase substrate (4-Methylumbelliferyl $\beta$-D-glucopyranoside, MilliporeSigma) in citrate-phosphate buffer, $\mathrm{pH} 4.6$ containing final concentrations of $1 \%$ bovine serum albumin, $0.25 \%$ triton $\mathrm{X}-100,0.25 \%$ taurocholic acid, and $1 \mathrm{mM}$ EDTA [40]. The reactions proceeded for $1 \mathrm{~h}$ at 
$37{ }^{\circ} \mathrm{C}$ and stopped by addition of $0.4 \mathrm{M}$ glycine, $\mathrm{pH} 10.8$. Specific GCase activity was confirmed for each sample by subtracting the activity of wells containing $0.18 \mathrm{mM}$ conduritol $\beta$-epoxide (Enzo Life Sciences), a GCase inhibitor. All assays were carried out in white, opaque 96-well plates and read on a Synergy 2 plate reader (Biotek Instruments) with an excitation wavelength of $360 \mathrm{~nm}$ and an emission wavelength of $440 \mathrm{~nm}$. The amount of methylumbelliferone freed during the reaction was quantitated relative to a standard curve of methylumbelliferone (4-MU) run on each plate, and results were calculated as nmol 4-MU/hour/mg protein.

\section{PNGase digestion}

Frontal cortical lysates prepared as described above were incubated overnight at $37^{\circ} \mathrm{C}$ with PNGase F (New England Biolabs) according the manufacturer's instructions. $50 \mu \mathrm{g}$ of protein were included in each reaction. For each sample, a control tube was incubated without PNGase F enzyme. After incubation, the samples were diluted with $4 \mathrm{X}$ LDS sample buffer (ThermoFisher Scientific) and 10X Bolt sample reducing agent (ThermoFisher Scientific) and heated for $10 \mathrm{~min}$ at $70{ }^{\circ} \mathrm{C}$ before analysis by western blot as described above.

\section{Statistics}

Tmem106b, progranulin, and LAMP-1 levels, as well as HexA, GCase, and Gusb activity in $\mathrm{Grn}^{+/-}:{\text {Tmem } 106 b^{+/-}}^{-1}$ mice (Figs. 1 and 4), were analyzed by two-way ANOVA with factors of Tmem106b and Grn. Significant main effects or interactions were followed by Tukey's post-hoc test. HexA and GCase activity and levels from aged $\mathrm{Grn}^{+/-}$mice (Fig. 3c-f) were analyzed by $t$ test. LAMP-1 levels and HexA, GCase, and Gusb activity in Tmem106b ${ }^{-1-}$ mice (Fig. 5), as well as HexA activity and levels for young mice (Fig. 3a, b) were analyzed by one-way ANOVA, and a significant genotype effect was followed by Dunnett's post-hoc test to compare heterozygous and homozygous knockout mice to wild-type. The number of wins per group in the tube test (Fig. 2a, c, e, g) was analyzed by binomial test to compare the observed vs. expected outcome, with the expected outcome set at 50\% wins per group. The winning percentage in the tube test (Fig. $2 \mathrm{~b}, \mathrm{~d}, \mathrm{f}, \mathrm{h}$ ) was analyzed by

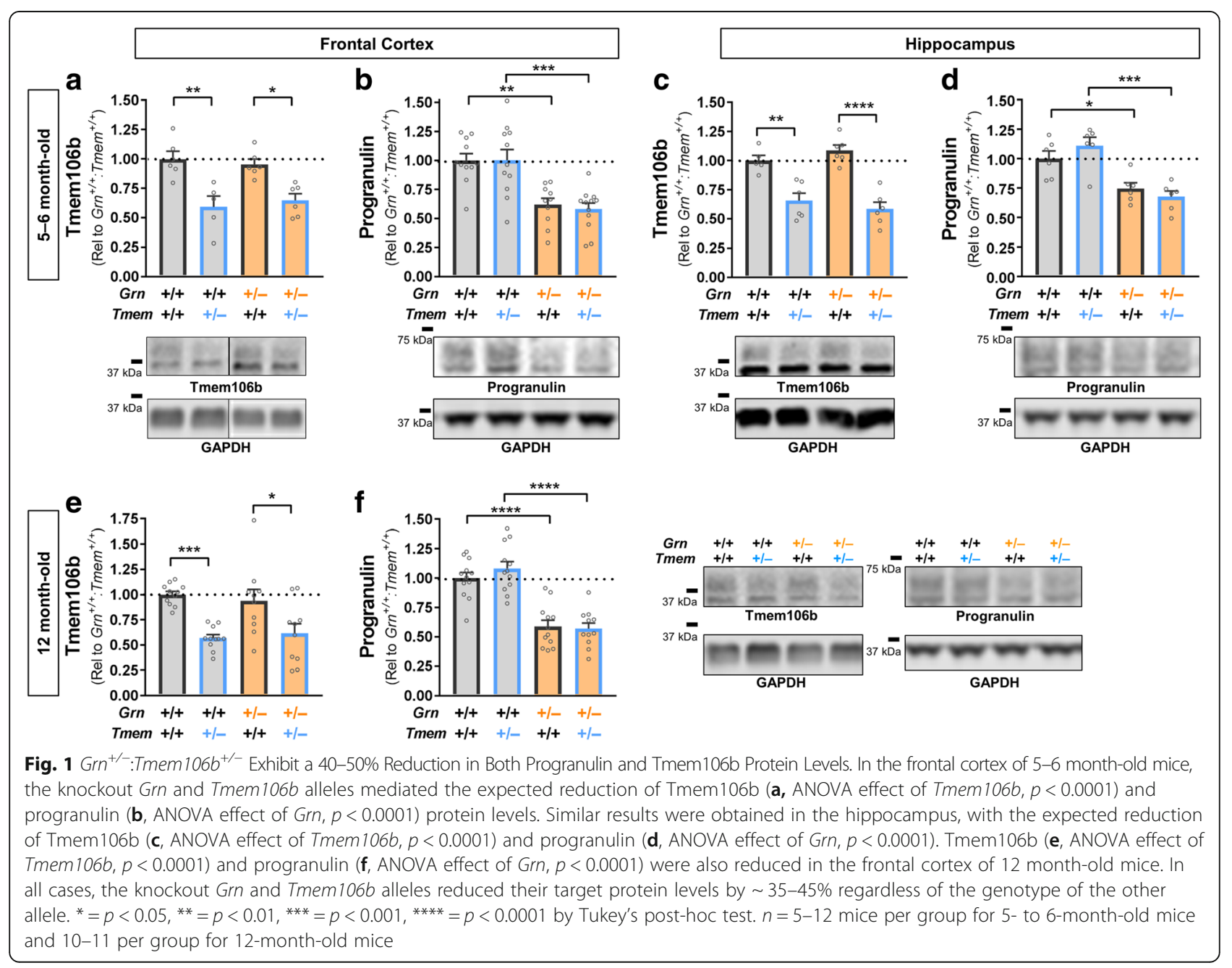




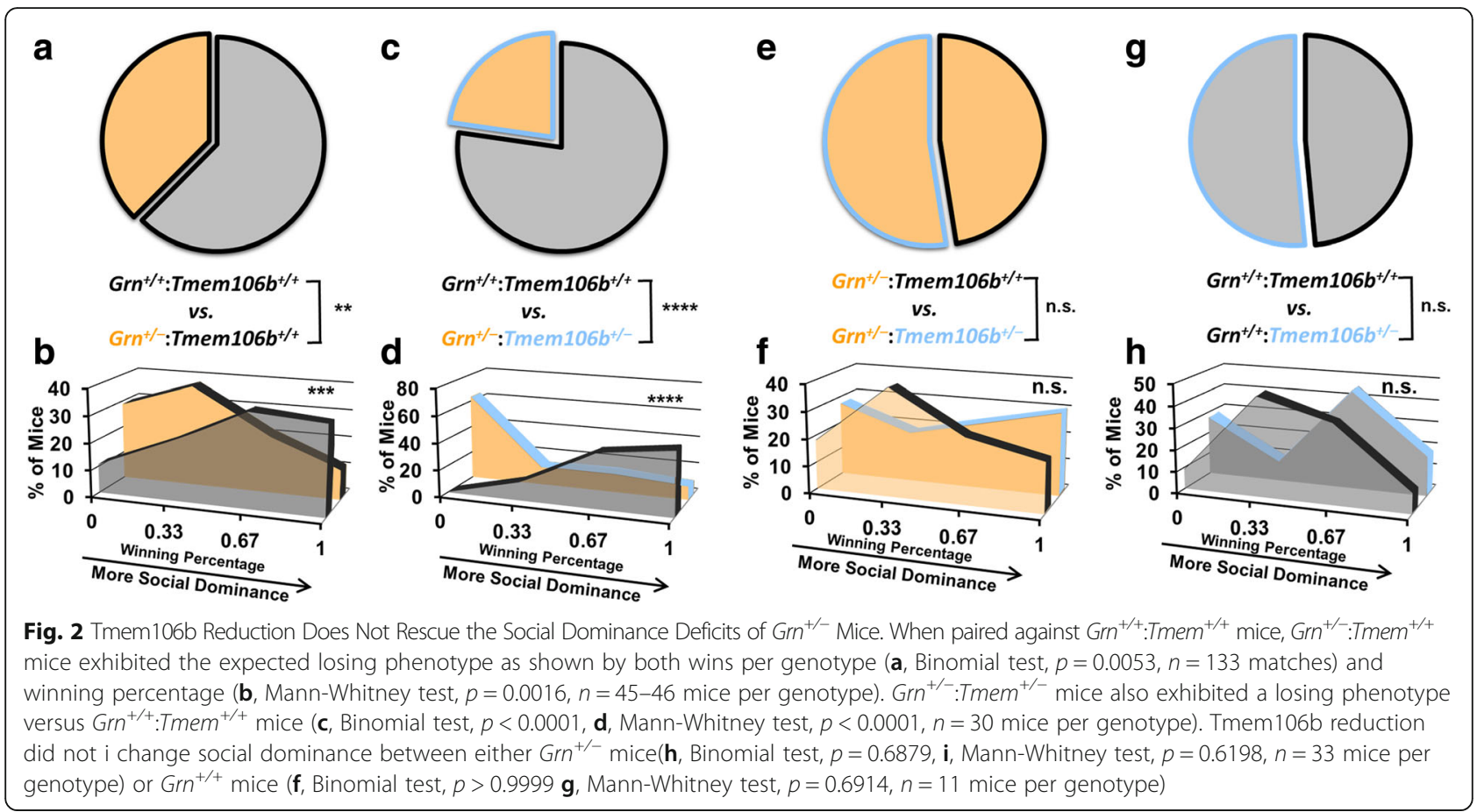

Mann-Whitney test. Two-tailed $p$ values were calculated for all analyses, with $\alpha$ set at 0.05 . All analyses were conducted with GraphPad Prism 7 (GraphPad Software).

\section{Results}

$\mathrm{Grn}^{+/-}: \mathrm{Tmem}^{106 \mathrm{~b}^{+/-}}$mice exhibit a similar reduction in cortical and hippocampal progranulin and Tmem106b protein levels

After generating $\mathrm{Grn}^{+/-}:$Tmem106b $\mathrm{b}^{+/-}$mice, we measured progranulin and Tmem106b levels in the frontal cortex and hippocampus of 5- to 6-month-old mice. We observed a similar reduction of Tmem106b levels $(\sim 35-45 \%)$ in Tmem106b ${ }^{+/-}$mice regardless of Grn genotype (Fig. 1a, c), and a similar reduction in progranulin levels $(\sim 35-45 \%)$ in $\mathrm{Grn}^{+/-}$mice regardless of Tmem106b genotype (Fig. 1b, d). Similarly, the 12-month-old mice used for behavioral studies described below exhibited reduction of progranulin and Tmem106b without signs of interaction between the two knockout alleles (Fig. 1e, f). We observed no effect of Tmem106b genotype on progranulin levels, and no effect of Grn genotype on Tmem106b levels.

\section{Tmem $106 \mathrm{~b}$ reduction does not rescue the low social} dominance phenotype of $\mathrm{Grn}^{+/-}$mice

To test the hypothesis that Tmem $106 \mathrm{~b}$ reduction may rescue deficits induced by progranulin haploinsufficiency,

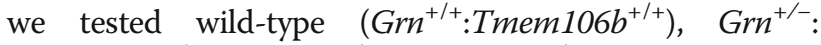
Tmem106b $b^{+/+}$, and $\mathrm{Grn}^{+/-}:$Tmem106b ${ }^{+/-}$mice in the tube test for social dominance at age 11-12 months. $\mathrm{Grn}^{+/-}$ mice develop a stable low dominance phenotype in this task after 9 months of age, making it a useful behavioral screen for social deficits induced by progranulin insufficiency [32]. When tested against wild-type mice, $\mathrm{Grn}^{+/-}: \mathrm{Tmem}_{106 b^{+/+}}$ mice exhibited a low dominance phenotype of a similar magnitude as previously reported (Fig. 2a, b) [32]. Tmem106b reduction did not correct this phenotype, as $\mathrm{Grn}^{+/-}: \mathrm{Tmem}_{106 b^{+/-}}$mice also exhibited a low dominance phenotype against wild-type mice (Fig. 2c, d). If anything, it appeared that the low dominance phenotype of $\mathrm{Grn}^{+/-}$: Tmem $106 b^{+/-}$mice might have even been worse than that of $\mathrm{Grn}^{+/-}:$Tmem $106 b^{+/+}$mice. To determine if this was a significant effect, i.e. whether Tmem106b reduction significantly reduced dominance in $\mathrm{Grn}^{+/-}$mice, we directly compared dominance in $\mathrm{Grn}^{+/-}:{\text {Tmem } 106 b^{+/+}}^{-1}$ mice and $\mathrm{Grn}^{+/-}:$Tmem $106 b^{+/-}$mice by pairing the groups against each other in the tube test. We observed no effect of Tmem106b genotype in this comparison (Fig. 2e, f). We similarly saw no effect of Tmem106b genotype on social dominance in $\mathrm{Grn}^{+/+}$mice (Fig. 2g, h). Based on these data, we conclude that Tmem $106 \mathrm{~b}$ reduction does not rescue the low dominance phenotype of $\mathrm{Grn}^{+/-}$mice, but does not worsen it either.

\section{$\mathrm{Grn}^{+/-}$mice exhibit elevated levels and activity of lysosomal enzymes in the frontal cortex}

We next asked if Tmem106b reduction had any effect on lysosomal abnormalities in $\mathrm{Grn}^{+/-}$mice. We previously reported that $\mathrm{Grn}^{+/-}$and $\mathrm{Grn}^{-1-}$ mice have increased levels of LAMP-1 in the frontal cortex at around 12 months of age [33]. To gain a more complete picture 


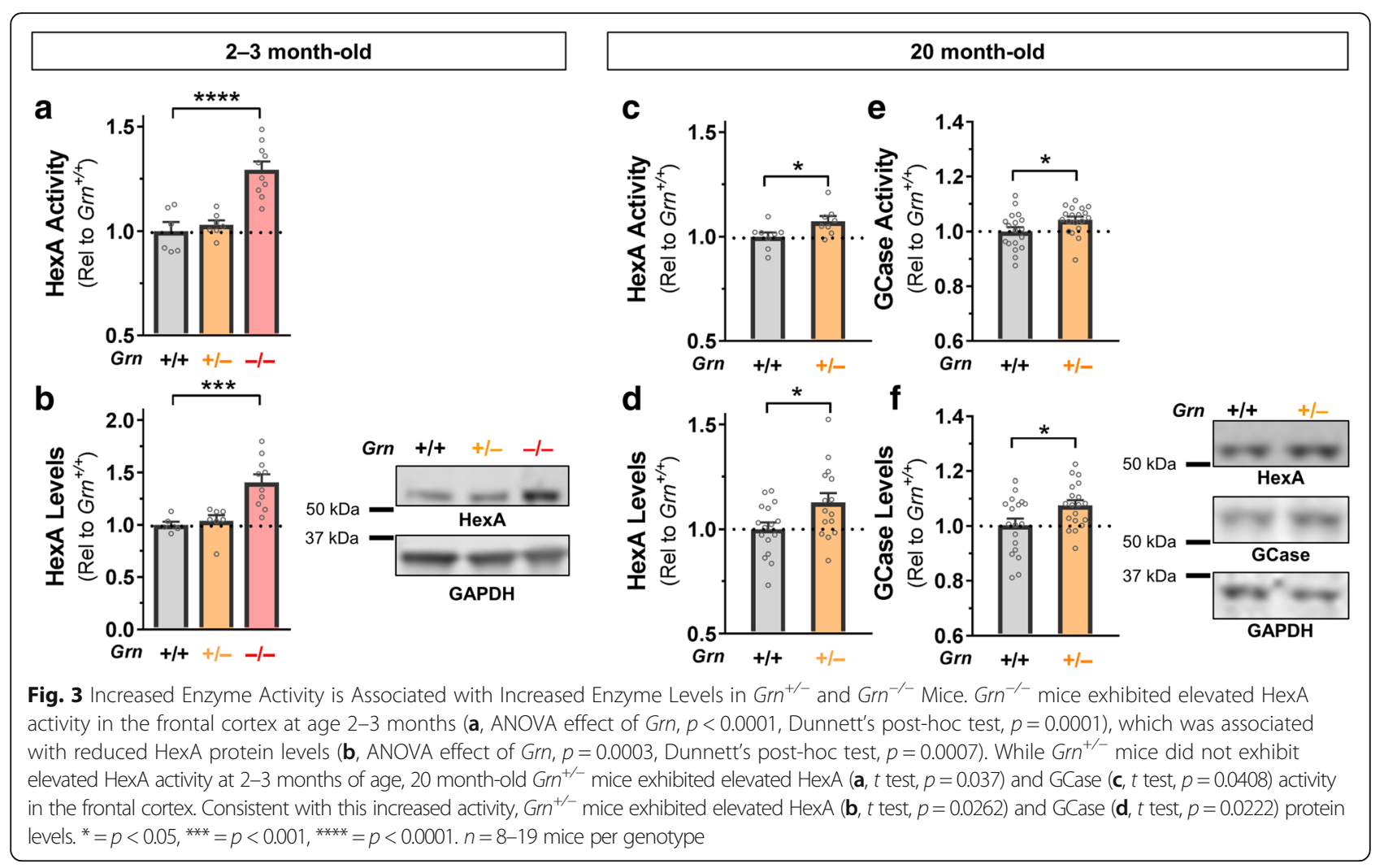

of the lysosomal abnormalities caused by progranulin insufficiency, we measured activity of the lysosomal enzyme $\beta$-hexosaminidase A (HexA), which has been reported to have increased expression and activity in $\mathrm{Grn}^{-/-}$mice $[35,41]$. Consistent with these prior reports, we observed elevated HexA activity and protein levels in the frontal cortex of $\mathrm{Grn}^{-/}$mice aged 2-3 months (Fig. 3a, b). Grn ${ }^{+/-}$mice did not have significantly elevated HexA at this age, though it is notable that they also lack detectable behavioral phenotypes at 2-3 months [32]. To determine if $\mathrm{Grn}^{+/-}$mice would exhibit lysosomal enzyme abnormalities at a more advanced age, we analyzed HexA activity and protein levels in the frontal cortex of 20-month-old wild-type and $\mathrm{Grn}^{+/-}$mice. These 20-month-old $\mathrm{Grn}^{+/-}$mice exhibited elevated HexA activity (Fig. 3c) and protein levels (Fig. 3d). To determine if similar changes could be observed in other lysosomal enzymes, we measured activity and protein levels of $\beta$-glucocerebrosidase (GCase), which is reported to be improperly trafficked in $\mathrm{Grn}^{-/-}$mice [42]. Similar to HexA, 20-month-old $\mathrm{Grn}^{+/-}$mice exhibited elevated GCase activity (Fig. 3e) and protein levels (Fig. 3f). Both HexA and GCase are glycosylated to facilitate their maturation and trafficking to the lysosome, and our western blots measured levels of the fully glycosylated proteins (Additional file 1: Figure S1). Along with the previously reported elevations in LAMP-1 levels [33], these novel lysosomal abnormalities show that brains from $\mathrm{Grn}^{+/-}$mice have elevated levels of lysosomal membrane proteins and mature lysosomal enzymes, which may be consistent with a compensatory response to an underlying lysosomal dysfunction.

Tmem 106b reduction does not normalize most lysosomal abnormalities of $\mathrm{Grn}^{+/-}$mice

We next tested whether Tmem106b reduction could normalize the elevated levels of LAMP-1 and activity of HexA and GCase in $\mathrm{Grn}^{+/-}$mice. Both HexA and GCase are involved in metabolism of glycosphingolipids [43], so we also measured activity of $\beta$-glucuronidase (Gusb), an enzyme involved in metabolism of glycosaminoglycans, to gain insight into a lysosomal enzyme involved in a different metabolic pathway and to provide a broader perspective on lysosomal enzyme changes in $\mathrm{Grn}^{+/-}$ mice. $\mathrm{Grn}^{+/-}$mice begin to show behavioral deficits around 6 months of age [31], and have well-established behavioral and lysosomal abnormalities by 12 months of age [31-33], so we measured LAMP-1 levels and the activity of HexA, GCase, and Gusb in frontal cortex samples from 5- to 6-month-old and 12-month-old mice to assess lysosomal abnormalities in $\mathrm{Grn}^{+/-}$mice in the early and established stages of abnormal behavior. 
While LAMP-1 and GCase activity remained unchanged in $\mathrm{Grn}^{+/-}$mice at 5- to 6-months of age (Fig. 4a, c), we observed significantly increased HexA (Fig. 4b) and Gusb (Fig. 4d) activity in the frontal cortex, showing that $\mathrm{Grn}^{+/}$ mice exhibit lysosomal abnormalities around the same age that behavioral deficits begin to develop [31]. In 12-month-old $\mathrm{Grn}^{+/-}$mice, we again observed increased HexA (Fig. 4f) and Gusb (Fig. 4h) activity, as well as increased LAMP-1 levels (Fig. 4e) and GCase activity (Fig. 4g). These data show that lysosomal abnormalities worsen in an age-dependent manner in $\mathrm{Grn}^{+/-}$frontal cortex along a parallel time-course with the ongoing development of behavior deficits [32]. We were able to measure protein levels of HexA and GCase by western blot in 12-month-old mice, but did not observe significant increases in $\mathrm{Grn}^{+/-}$mice (data not shown). The failure to detect a change in enzyme levels may be due to the less quantitative nature of western blot relative to fluorometric enzyme assay, and could also be due to ongoing increases in enzyme levels between 12 and 20 months of age in $\mathrm{Grn}^{+/-}$mice.

Tmem106b reduction failed to normalize the increased LAMP-1 levels (Fig. 4e), HexA activity (Fig. 4b, f), and
GCase activity (Fig. 4g) of $\mathrm{Grn}^{+/-}$mice, leading us to conclude that Tmem106b reduction does not normalize the lysosomal dysfunction of $\mathrm{Grn}^{+/-}$mice. However, Tmem106b reduction did compensate for the increased Gusb activity of $\mathrm{Grn}^{+/-}$mice at both 5-6 and 12 months of age (Fig. 4d, h). Tmem106b reduction also produced a trend for lowering Gusb activity in $\mathrm{Grn}^{+/+}$mice (Fig. 4d, h), potentially indicating that the normalization of Gusb activity in $\mathrm{Grn}^{+/-}:$Tmem $106 b^{+/-}$mice is due to independent suppression of Gusb activity by Tmem106b reduction instead of a correction of lysosomal dysfunction in $\mathrm{Grn}^{+/-}$ mice, i.e. "pseudonormalization".

\section{Tmem 106b reduction suppresses $\beta$-Glucuronidase activity independent of progranulin genotype}

To test whether suppression of Gusb activity by Tmem106b reduction, independently of progranulin, could explain the normalization of Gusb activity in $\mathrm{Grn}^{+/-}$:Tmem106b $b^{+/-}$ mice, we analyzed the previously described lysosomal markers in Tmem106b ${ }^{+/+}$, Tmem106b ${ }^{+/-}$, and Tmem106b ${ }^{-/-}$ mice on a $\mathrm{Grn}^{+/+}$background. As predicted, we observed a significant suppression $(\sim 20 \%)$ of Gusb activity in Tmem106b $^{+/-}$and Tmem106b ${ }^{-/-}$mice (Fig. 5d).

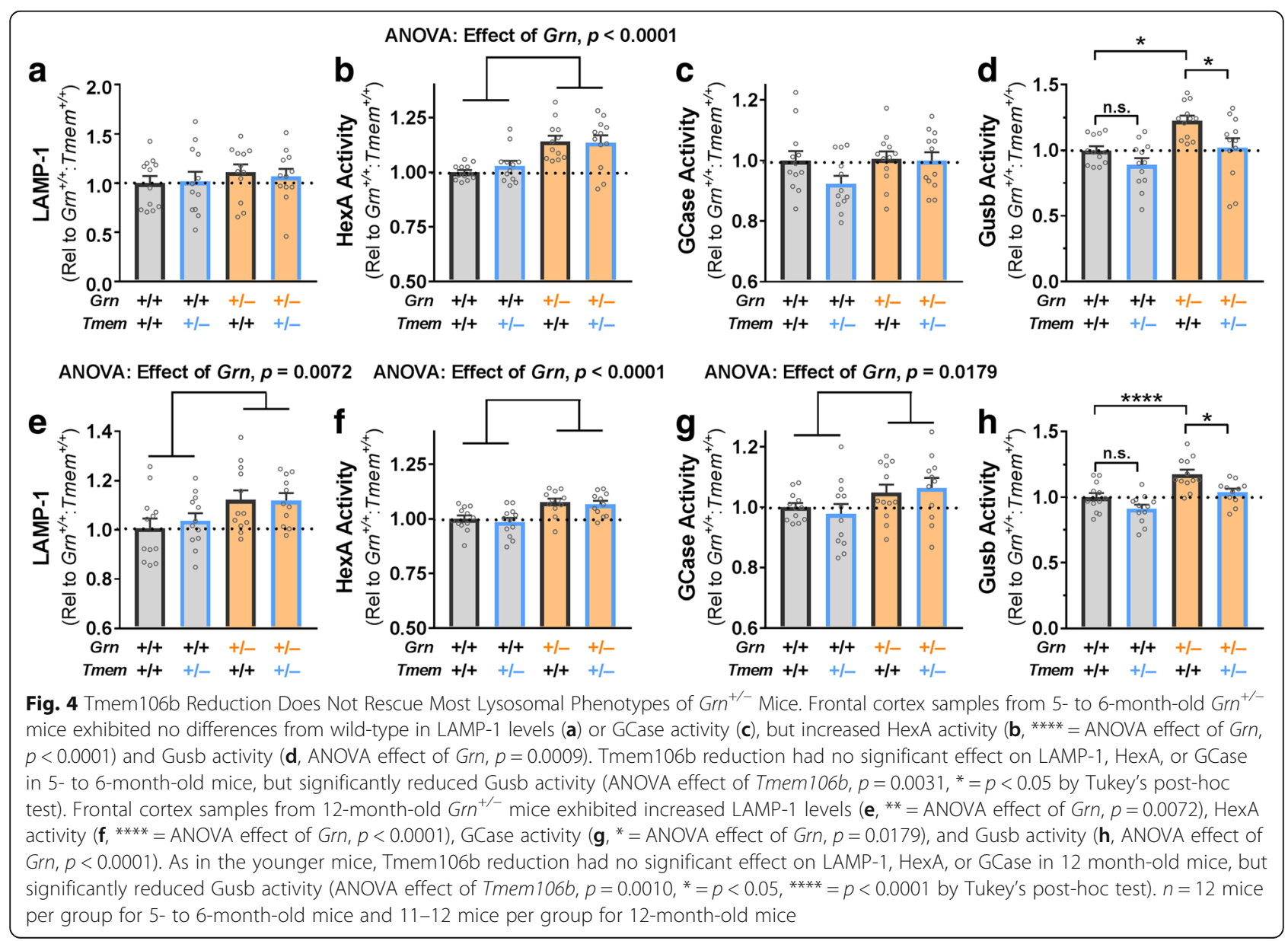


Tmem $106 b^{+/-}$and Tmem $106 b^{-/-}$mice did not exhibit significant decreases in LAMP-1 activity (Fig. 5a) or in GCase activity (Fig. 5c). Interestingly, Tmem106b ${ }^{+/-}$, but not Tmem $106 b^{-/-}$mice, exhibited a mild suppression ( 10\%) of HexA activity (Fig. 5b). This was somewhat unexpected, as $\mathrm{Grn}^{+/+}: \mathrm{Tmem}_{106 b^{+/}}$mice in our previous experiment did not have suppressed HexA activity (Fig. 4b, f). This discrepancy may be related to the age of the mice, as these mice were aged 2-5 months (average age around 4 months), while the experiments in Fig. 4 were done on older mice (average ages of around 6 and 12 months). HexA activity was not suppressed in Tmem $106 b^{-/}$mice, which has also been observed by others [35]. In previous studies with progranulin-insufficient mice, we have observed phenotypes in $\mathrm{Grn}^{+/-}$mice that were not present in $\mathrm{Grn}^{-/-}$mice, so these data might indicate mild, differential effects of partial Tmem106b reduction versus total deletion of Tmem106b. In summary, we conclude that Tmem106b reduction suppresses Gusb activity, but may also have a weaker, age-dependent suppressive effect on HexA activity.

\section{Discussion}

This study shows that an approximate 50\% reduction of Tmem106b levels is not sufficient to rescue behavioral and most lysosomal abnormalities in a $\mathrm{Grn}^{+/-}$mouse model of FTD. Partial Tmem106b reduction had measurable effects on lysosomal function, particularly Gusb activity, but this did not correct the other lysosomal abnormalities or the loss of social dominance seen in $\mathrm{Grn}^{+/-}$mice. These data suggest that the strong protective effects of certain TMEM106B SNPs in FTD-GRN may result from mechanisms apart from reduced TMEM106B levels.

This study was premised on consistently reproduced evidence that SNPs in TMEM106B modulate risk for FTD-TDP, with a particularly strong effect in GRN mutation carriers [10-16]. While the evidence that these TMEM106B SNPs protect against FTD in GRN carriers is strong, the mechanism by which they do so is not yet clear. As previously discussed, several lines of evidence indicate that protective alleles of these SNPs may reduce TMEM106B protein levels through either reduced expression of TMEM106B RNA or by enhanced degradation of TMEM106B protein $[10,11,13,17-19]$. We modeled this potentially protective TMEM106B reduction using Tmem $106 b^{+/-}$mice, which exhibited a $35-45 \%$ reduction in Tmem106b protein levels (Fig. 1a, c, f). We crossed $\mathrm{Tmem} 106 b^{+/-}$mice with $\mathrm{Grn}^{+/-}$mice to test the hypothesis that Tmem106b reduction could rescue deficits induced by progranulin insufficiency.

Tmem106b reduction failed to normalize the behavioral and most lysosomal deficits of $\mathrm{Grn}^{+/-}$mice, showing that partial Tmem106b reduction does not rescue most deficits induced by progranulin insufficiency in mice. In considering the implications of these results for TMEM106B/GRN interactions in humans, it is important to note the strengths and weaknesses of the mouse models used for this study. The gene-trap method used to knock out Tmem106b in this study leaves the first three exons of the Tmem106b gene in place [35], raising the potential for Tmem106b fragments to persist from the knockout allele. However, we did not detect any such fragments by western blot using an antibody recognizing the amino-terminal part of the protein that should be present in any such fragments, and we observed the expected loss of nearly $50 \%$ of Tmem106b protein levels (Fig. 1). Nonetheless, the potential presence of Tmem106b fragments from the knockout allele of this Tmem106b mouse model raises a need for future studies with Tmem106b knockout models featuring complete deletion of the Tmem106b coding region.

We consider the partial reduction of both Tmem106b and progranulin through the use of $T m e m 106 b^{+/-}$and $\mathrm{Grn}^{+/-}$mice to be a strength of this study, as the heterozygous knockouts model the partial reduction in gene expression caused by both protective TMEM106B SNPs and GRN haploinsufficiency. Protective TMEM106B SNPs
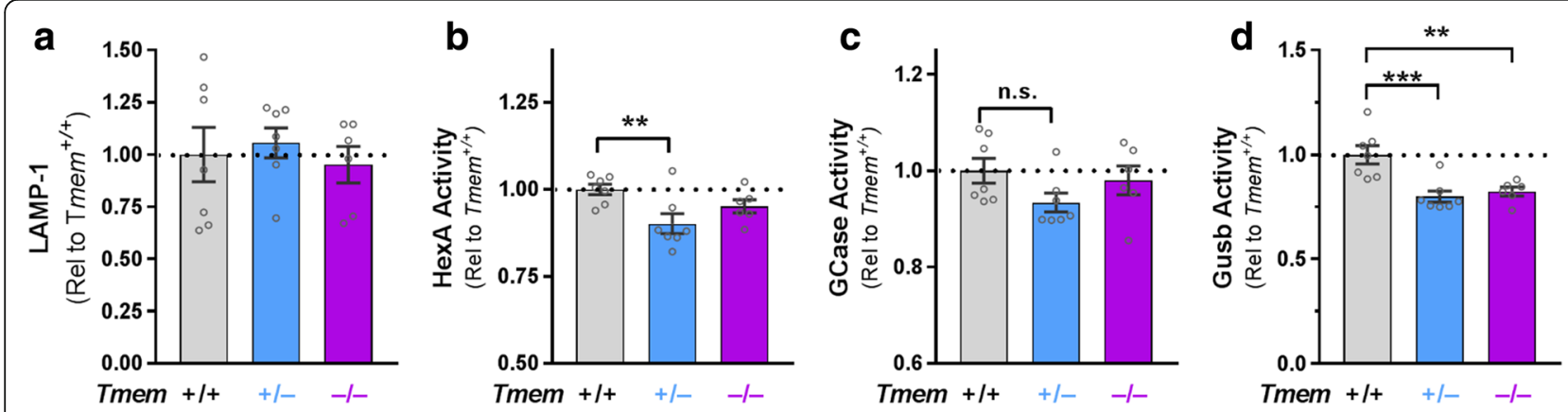

Fig. 5 Tmem106b Reduction Suppresses Activity of $\beta$-Glucuronidase. Tmem106b reduction had no significant effect on LAMP-1 levels (a) or GCase activity (c) in the frontal cortex of 2- to 5-month-old mice. HexA activity was significantly reduced in Tmem 106b ${ }^{+/-}$mice (b, ANOVA effect of

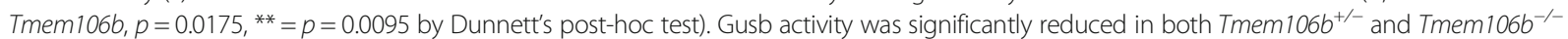
mice (d, ANOVA effect of Tmem 106b, $p=0.0007,{ }^{* *}=p<0.01,{ }^{* * *}=p<0.001$ by Dunnett's post-hoc test). $n=6-7$ mice per genotype 
are associated with reduced TMEM106B mRNA or protein levels, but not total loss of TMEM106B [10, 17, 19]. Tmem $106 b^{+/-}$mice thus model the partial TMEM106B reduction mediated by protective SNPs, although they may not achieve the same magnitude of reduction as the TMEM106B SNPs. FTD-GRN is caused by heterozygous mutations, while individuals homozygous for pathogenic GRN mutations develop neuronal ceroid lipofuscinosis instead of FTD $[22,23]$, making $\mathrm{Grn}^{+/-}$mice a closer genetic model of human FTD-GRN than $\mathrm{Grn}^{-/-}$mice. $\mathrm{Grn}^{+/-}$provide an accurate model of most of the common FTD-causing GRN mutations, which reduce progranulin levels through nonsense-mediated decay and cause a complete loss of protein from the mutant allele [6]. However, other GRN mutations may produce some dysfunctional protein product $[44,45]$. It may therefore be of interest to study the effects of Tmem106b reduction in knock-in models of GRN mutations that produce some protein product.

Although the lysosomal abnormalities of $\mathrm{Grn}^{+/-}$mice are much milder than those of $\mathrm{Grn}^{-/-}$mice, $\mathrm{Grn}^{+/-}$mice have interesting, likely disease-relevant abnormalities, and this study adds to the known lysosomal changes of $\mathrm{Grn}^{+/-}$mice. $\mathrm{Grn}^{+/-}$mice have well-documented behavioral abnormalities [31-33], but the cellular basis for these abnormalities has remained elusive. Observation of lysosomal abnormalities in brains from FTD patients with GRN mutations has led to the hypothesis that progranulin haploinsufficiency impairs lysosomal function, albeit less severely than complete progranulin deficiency, and that FTD-GRN may be on a continuum of lysosomal dysfunction with NCL due to GRN mutations [21, 25]. These novel lysosomal abnormalities in $\mathrm{Grn}^{+/-}$mice (Fig. 3), as well as previous reports of increased LAMP-1 levels [33] and the more dramatic lysosomal abnormalities of $\mathrm{Grn}^{-/-}$ mice $[21,35,41,46]$, indicate that mice may model the gene-dose effect of progranulin insufficiency on lysosomal function. These lysosomal changes may be related to the behavioral deficits of $\mathrm{Grn}^{+/-}$mice, as they appear in a roughly similar time-course [32] and are normalized along with social dominance deficits by boosting progranulin levels with an AAV vector [33].The enzymes with increased activity in $\mathrm{Grn}^{+/-}$mice (HexA, GCase, and Gusb) are all part of the CLEAR (coordinated lysosomal expression and regulation) network of lysosomal genes that is activated in response to lysosomal dysfunction $[47,48]$. Given that the increased activity of HexA and GCase in $\mathrm{Grn}^{+/-}$mice is associated with increased levels of these enzymes, these increases are likely to be compensatory responses to as-yet uncharacterized lysosomal deficits induced by progranulin insufficiency (Fig. 6). In this context, it is unsurprising that normalization of Gusb activity alone failed to alter the social dominance phenotype of $\mathrm{Grn}^{+/-}$mice.

The counteraction of some lysosomal abnormalities in progranulin-insufficient mice by Tmem106b reduction (Fig. 4) is consistent with a recent study in which

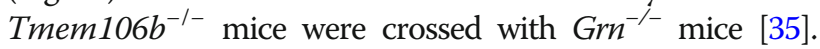
This study reported opposing effects of Grn and Tmem106b knockout on expression of many lysosomal genes, with Grn knockout elevating expression and Tmem106b knockout reducing expression [35]. For lysosomal enzymes affected by both Grn and Tmem106b, their opposing effects resulted in enzyme activity that did not differ from wild-type in $\mathrm{Grn}^{-1-}$ :

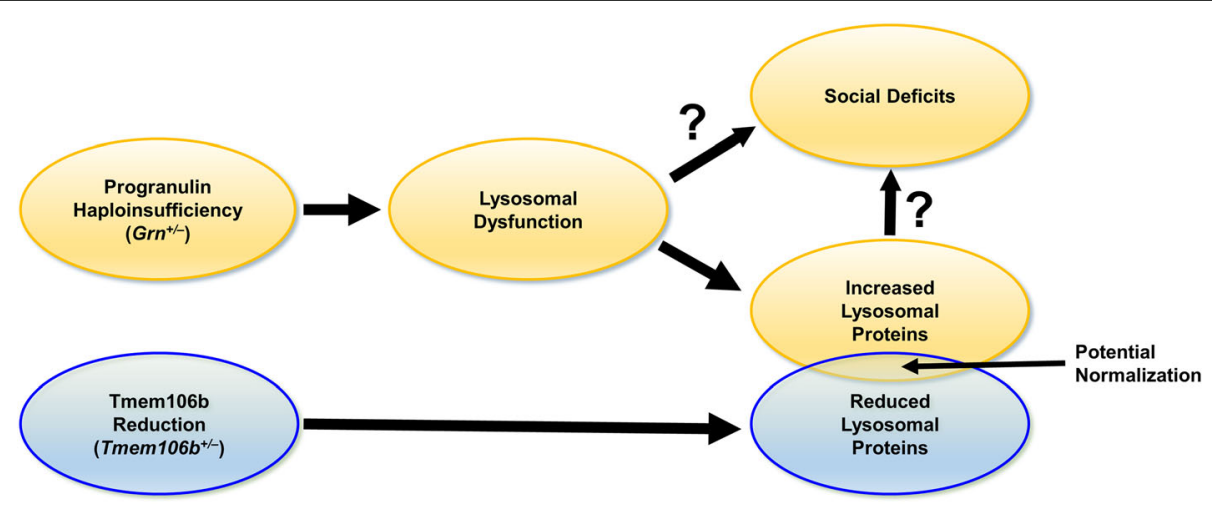

Fig. 6 Proposed Model of the Effects of Progranulin and Tmem106b in Grn ${ }^{+/-}:$Tmem 106b ${ }^{+/-}$Mice. The current study and others [35], indicate that Tmem106b reduction in progranulin insufficient mice normalizes some lysosomal abnormalities induced by progranulin insufficiency, but fails to rescue the most abnormalities caused by progranulin insufficiency. In this model, progranulin insufficiency causes lysosomal dysfunction, which may then cause the social deficits of $\mathrm{Grn}^{+/-}$mice. In addition, levels of many lysosomal proteins are increased, probably as a result of increased lysosomal gene expression via transcription factor EB. This increase in lysosomal proteins may also contribute to social behavior deficits, or may be a parallel phenomenon resulting from the underlying lysosomal dysfunction. In contrast, Tmem106b reduction suppresses the expression of many lysosomal genes (Figs. 3, 4 and [35]). Genes affected by both progranulin insufficiency and Tmem106b reduction, such as Gusb (Fig. 3d, h), may have normalized activity in $\mathrm{Grn}^{+/}: \mathrm{Tmem} 106 b^{+/-}$mice, but lysosomal dysfunction and social deficits remain intact in $\mathrm{Grn}^{+/-}: \mathrm{Tmem}^{106 b^{+/-}}$mice 
Tmem106b ${ }^{-1-}$ mice [35]. However, other lysosomal enzymes maintained elevated activity in $\mathrm{Grn}^{-/-}: \mathrm{Tmem}_{106 b^{-/-}}$ mice [35]. We obtained similar data, in which $\beta$-glucuronidase activity was suppressed in $T m e m 106 b^{+/-}$mice regardless of Grn genotype (Figs. 4, 5). Also, while complete Tmem106b knockout prevented open field, plus maze, and retinal abnormalities in $\mathrm{Grr}^{-/-}$mice [35]; in both the prior study and ours, Tmem106b reduction failed to correct other key phenotypes, including lipofuscinosis and neuroinflammation in $\mathrm{Grn}^{-1-}$ mice [35] and social deficits in $\mathrm{Grn}^{+/-}$mice (Fig. 2). Taken together, these data indicate that Tmem106b reduction may counteract some of the effects of progranulin insufficiency on lysosomal function, but does not completely rescue the lysosomal dysfunction induced by progranulin insufficiency (Fig. 6). Conversely, transgenic overexpression of TMEM106B in $\mathrm{Grn}^{-1-}$ mice worsens lipofuscinosis and lysosomal enlargement in $\mathrm{Grn}^{-1-}$ mice, which is consistent with the deleterious effects of TMEM106B overexpression on lysosomal function in cell culture $[17,20,26-28,34]$.

\section{Conclusions}

While further study is needed, the data obtained from this study suggests that reduction of TMEM106B levels may not be the primary mechanism by which TMEM106B SNPs protect against FTD due to GRN mutations. Other aspects of these SNPs in linkage disequilibrium, such as the S/T coding variant, may alter TMEM106B function or interaction with other lysosomal proteins. It is also possible that human TMEM106B and progranulin interact in ways not modeled by mice, making human cell lines a potential model for further research. Such studies would complement the strength of mouse models in uncovering the molecular mechanisms that arise the aging brain. Given the clear protective effect of TMEM106B SNPs against multiple FTD subtypes and the emerging role of TMEM106B in aging and other neurodegenerative diseases [10-18, 49, 50], further study of TMEM106B's effects in the brain are an important area for future research.

\section{Additional file}

Additional file 1: Figure S1. Measurement of Glycosylated HexA and GCase by Western Blot. The western blots in fig. 3 measured the glycosylated forms of HexA and GCase, which was confirmed by loss of these bands after digestion with the glycosidase PNGase F. The glycosylated forms of HexA and GCase are labeled with black arrows, the unglycosylated forms with gray arrows, and nonspecific bands with asterisks. (DOCX 195 kb)

\section{Abbreviations}

FTD: frontotemporal dementia; GCase: $\beta$-glucocerebrosidase; GRN: progranulin; Gusb: $\beta$-glucuronidase; HexA: $\beta$-hexosaminidase A; SNP: single nucleotide polymorphism

\section{Acknowledgements}

This work was supported by the Consortium for FTD Research and the Bluefield Project to Cure FTD, the National Institute of Neurological Disorders and Stroke (R01NS075487, P30NS047466 and R35NS097261), and the National Institute on Aging (K99AG056597). We thank James Black, Miriam Roberson, and Ralph B. Perkerson III for help with mouse breeding and colony maintenance, and John Shacka for assistance with lysosomal enzyme assays. Behavior experiments were performed in the Evelyn F. McKnight Brain Institute Behavior Core Facility at the University of Alabama at Birmingham.

\section{Funding}

This work was supported by the Consortium for FTD Research and the Bluefield Project to Cure FTD, the National Institute of Neurological Disorders and Stroke (R01NS075487, P30NS047466 and R35NS097261), and the National Institute on Aging (K99AG056597).

\section{Availability of data and materials}

All data generated or analyzed during this study are included in this published article.

\section{Authors' contributions}

All authors participated in design of experiments and contributed to the manuscript. AMN, XZ, and RR provided the Tmem $106 b^{+/}$mouse line. AEA and EDR crossed Tmem $106 b^{+/-}$mice with $\mathrm{Grn}^{+/-}$mice and analyzed behavior and lysosomal phenotypes. All authors read and approved the final manuscript.

Ethics approval and consent to participate

All experiments were approved by the Institutional Animal Care and Use Committee at the University of Alabama at Birmingham.

Competing interests

The authors declare that they have no competing interests.

\section{Publisher's Note}

Springer Nature remains neutral with regard to jurisdictional claims in published maps and institutional affiliations.

Received: 15 March 2018 Accepted: 25 May 2018

Published online: 22 June 2018

\section{References}

1. Bateman A, Bennett HP. Granulins: the structure and function of an emerging family of growth factors. J Endocrinol. 1998;158:145-51.

2. Eriksen JL, Mackenzie IR. Progranulin: normal function and role in neurodegeneration. J Neurochem. 2008;104:287-97.

3. Nicholson AM, Gass J, Petrucelli L, Rademakers R. Progranulin axis and recent developments in frontotemporal lobar degeneration. Alzheimers Res Ther. 2012;4:4.

4. Nguyen AD, Nguyen TA, Martens LH, Mitic LL, Farese RV Jr. Progranulin: at the interface of neurodegenerative and metabolic diseases. Trends Endocrinol Metab. 2013:24:597-606.

5. Petkau TL, Leavitt BR. Progranulin in neurodegenerative disease. Trends Neurosci. 2014:37:388-98.

6. Baker M, Mackenzie IR, Pickering-Brown SM, Gass J, Rademakers R, Lindholm C, Snowden J, Adamson J, Sadovnick AD, Rollinson S, et al. Mutations in progranulin cause tau-negative frontotemporal dementia linked to chromosome 17. Nature. 2006;442:916-9.

7. Cruts M, Giiselinck I, van der Zee J, Engelborghs S, Wils H, Pirici D, Rademakers R, Vandenberghe R, Dermaut B, Martin JJ, et al. Null mutations in progranulin cause ubiquitin-positive frontotemporal dementia linked to chromosome 17q21. Nature. 2006;442:920-4.

8. Gass J, Cannon A, Mackenzie IR, Boeve B, Baker M, Adamson J, Crook R, Melquist S, Kuntz $K$, Petersen $R$, et al. Mutations in progranulin are a major cause of ubiquitinpositive frontotemporal lobar degeneration. Hum Mol Genet. 2006;15:2988-3001.

9. Finch N, Baker M, Crook R, Swanson K, Kuntz K, Surtees R, Bisceglio G, Rovelet-Lecrux A, Boeve B, Petersen RC, et al. Plasma progranulin levels predict progranulin mutation status in frontotemporal dementia patients and asymptomatic family members. Brain. 2009;132:583-91.

10. Van Deerlin VM, Sleiman PM, Martinez-Lage M, Chen-Plotkin A, Wang LS, Graff-Radford NR, Dickson DW, Rademakers R, Boeve BF, Grossman M, et al. Common variants at 7p21 are associated with frontotemporal lobar degeneration with TDP-43 inclusions. Nat Genet. 2010;42:234-9.

11. van der Zee J, Van Langenhove $T$, Kleinberger $G$, Sleegers $K$, Engelborghs $S$, Vandenberghe R, Santens P, Van den Broeck M, Joris G, Brys J, et al. 
TMEM106B is associated with frontotemporal lobar degeneration in a clinically diagnosed patient cohort. Brain. 2011;134:808-15.

12. Finch N, Carrasquillo MM, Baker M, Rutherford NJ, Coppola G, DejesusHernandez M, Crook R, Hunter T, Ghidoni R, Benussi L, et al. TMEM106B regulates progranulin levels and the penetrance of FTLD in GRN mutation carriers. Neurology. 2011;76:467-74.

13. Cruchaga C, Graff C, Chiang HH, Wang J, Hinrichs AL, Spiegel N, Bertelsen S, Mayo K, Norton JB, Morris JC, Goate A. Association of TMEM106B gene polymorphism with age at onset in granulin mutation carriers and plasma granulin protein levels. Arch Neurol. 2011;68:581-6.

14. Johnson SC, Christian BT, Okonkwo OC, Oh JM, Harding S, Xu G, Hillmer AT, Wooten DW, Murali D, Barnhart TE, et al. Amyloid burden and neural function in people at risk for Alzheimer's disease. Neurobiol Aging. 2014;35:576-84.

15. van Blitterswijk M, Mullen B, Nicholson AM, Bieniek KF, Heckman MG, Baker MC, DeJesus-Hernandez M, Finch NA, Brown PH, Murray ME, et al. TMEM106B protects C9ORF72 expansion carriers against frontotemporal dementia. Acta Neuropathol. 2014;127:397-406.

16. Lattante S, Le Ber I, Galimberti D, Serpente M, Rivaud-Pechoux S, Camuzat A, Clot F, Fenoglio C, French research network on FTD, Ftd ALS, et al. Defining the association of TMEM106B variants among frontotemporal lobar degeneration patients with GRN mutations and C9orf72 repeat expansions. Neurobiol Aging. 2014;35:2658 e2651-5.

17. Gallagher MD, Posavi M, Huang P, Unger TL, Berlyand Y, Gruenewald AL, Chesi A, Manduchi E, Wells AD, Grant SFA, et al. A dementia-associated risk variant near TMEM106B alters chromatin architecture and gene expression. Am J Hum Genet. 2017;101:643-63.

18. Yu L, De Jager PL, Yang J, Trojanowski JQ, Bennett DA, Schneider JA. The TMEM106B locus and TDP-43 pathology in older persons without FTLD. Neurology. 2015;84:927-34

19. Nicholson AM, Finch NA, Wojtas A, Baker MC, Perkerson RB 3rd, CastanedesCasey M, Rousseau L, Benussi L, Binetti G, Ghidoni R, et al. TMEM106B p. T185S regulates TMEM106B protein levels: implications for frontotemporal dementia. J Neurochem. 2013;126:781-91.

20. Chen-Plotkin AS, Unger TL, Gallagher MD, Bill E, Kwong LK, Volpicelli-Daley L, Busch Jl, Akle S, Grossman M, Van Deerlin V, et al. TMEM106B, the risk gene for frontotemporal dementia, is regulated by the microRNA-132/212 cluster and affects progranulin pathways. J Neurosci. 2012;32:11213-27.

21. Götzl JK, Mori K, Damme M, Fellerer K, Tahirovic S, Kleinberger G, Janssens J, van der Zee J, Lang CM, Kremmer E, et al. Common pathobiochemical hallmarks of progranulin-associated frontotemporal lobar degeneration and neuronal ceroid lipofuscinosis. Acta Neuropathol. 2014;127:845-60.

22. Smith KR, Damiano J, Franceschetti S, Carpenter S, Canafoglia L, Morbin M, Rossi G, Pareyson D, Mole SE, Staropoli JF, et al. Strikingly different clinicopathological phenotypes determined by progranulin-mutation dosage. Am J Hum Genet. 2012;90:1102-7.

23. Almeida MR, Macario MC, Ramos L, Baldeiras I, Ribeiro MH, Santana I. Portuguese family with the co-occurrence of frontotemporal lobar degeneration and neuronal ceroid lipofuscinosis phenotypes due to progranulin gene mutation. Neurobiol Aging. 2016;41:200 e201-5.

24. Ward ME, Taubes A, Chen R, Miller BL, Sephton CF, Gelfand JM, Minami S, Boscardin J, Martens LH, Seeley WW, et al. Early retinal neurodegeneration and impaired ran-mediated nuclear import of TDP-43 in progranulindeficient FTLD. J Exp Med. 2014;211:1937-45.

25. Ward ME, Chen R, Huang HY, Ludwig C, Telpoukhovskaia M, Taubes A, Boudin H, Minami SS, Reichert M, Albrecht $P$, et al. Individuals with progranulin haploinsufficiency exhibit features of neuronal ceroid lipofuscinosis. Sci Transl Med. 2017;9

26. Reiman EM, Quiroz YT, Fleisher AS, Chen K, Velez-Pardo C, Jimenez-Del-Rio M, Fagan AM, Shah AR, Alvarez S, Arbelaez A, et al. Brain imaging and fluid biomarker analysis in young adults at genetic risk for autosomal dominant Alzheimer's disease in the presenilin 1 E280A kindred: a case-control study. Lancet Neurol. 2012;11:1048-56.

27. Brady OA, Zheng $Y$, Murphy $K$, Huang M, Hu F. The frontotemporal lobar degeneration risk factor, TMEM106B, regulates lysosomal morphology and function. Hum Mol Genet. 2013;22:685-95.

28. Stagi M, Klein ZA, Gould TJ, Bewersdorf J, Strittmatter SM. Lysosome size, motility and stress response regulated by fronto-temporal dementia modifier TMEM106B. Mol Cell Neurosci. 2014;61:226-40.

29. Capell A, Liebscher S, Fellerer K, Brouwers N, Willem M, Lammich S, Gijselinck I, Bittner T, Carlson AM, Sasse F, et al. Rescue of progranulin deficiency associated with frontotemporal lobar degeneration by alkalizing reagents and inhibition of vacuolar ATPase. J Neurosci. 2011;31:1885-94.

30. Schwenk BM, Lang CM, Hogl S, Tahirovic S, Orozco D, Rentzsch K, Lichtenthaler SF, Hoogenraad CC, Capell A, Haass C, Edbauer D. The FTLD risk factor TMEM106B and MAP6 control dendritic trafficking of lysosomes. EMBO J. 2014;33:450-67.

31. Filiano AJ, Martens LH, Young AH, Warmus BA, Zhou P, Diaz-Ramirez G, Jiao J, Zhang Z, Huang EJ, Gao FB, et al. Dissociation of frontotemporal dementia-related deficits and neuroinflammation in progranulin haploinsufficient mice. J Neurosci. 2013;33:5352-61.

32. Arrant AE, Filiano AJ, Warmus BA, Hall AM, Roberson ED. Progranulin haploinsufficiency causes biphasic social dominance abnormalities in the tube test. Genes Brain Behav. 2016;15:588-603.

33. Arrant $A E$, Filiano $A J$, Unger DE, Young AH, Roberson ED. Restoring neuronal progranulin reverses deficits in a mouse model of frontotemporal dementia. Brain. 2017;140:1447-65.

34. Zhou X, Sun L, Brady OA, Murphy KA, Hu F. Elevated TMEM106B levels exaggerate lipofuscin accumulation and lysosomal dysfunction in aged mice with progranulin deficiency. Acta neuropathologica communications. 2017:5:9.

35. Klein ZA, Takahashi H, Ma M, Stagi M, Zhou M, Lam T, Strittmatter SM. Loss of TMEM106B ameliorates lysosomal and frontotemporal dementia-related phenotypes in Progranulin-deficient mice. Neuron. 2017;95:281-96. e286

36. Martens LH, Zhang J, Barmada SJ, Zhou P, Kamiya S, Sun B, Min S-W, Gan L, Finkbeiner S, Huang EJ, Robert V, Farese J. Progranulin deficiency promotes neuroinflammation and neuron loss following toxin-induced injury. J Clin Invest. 2012;122:3955-9.

37. Evers BM, Rodriguez-Navas C, Tesla RJ, Prange-Kiel J, Wasser CR, Yoo KS, McDonald J, Cenik B, Ravenscroft TA, Plattner F, et al. Lipidomic and transcriptomic basis of lysosomal dysfunction in Progranulin deficiency. Cell Rep. 2017;20:2565-74.

38. Dunn SD. Effects of the modification of transfer buffer composition and the renaturation of proteins in gels on the recognition of proteins on western blots by monoclonal antibodies. Anal Biochem. 1986;157:144-53.

39. Wendeler M, Sandhoff K. Hexosaminidase assays. Glycoconj J. 2009;26:945-52.

40. Nelson MP, Boutin M, Tse TE, Lu H, Haley ED, Ouyang X, Zhang J, Auray-Blais C, Shacka JJ. The lysosomal enzyme alpha-galactosidase a is deficient in Parkinson's disease brain in association with the pathologic accumulation of alpha-synuclein. Neurobiol Dis. 2018;110:68-81.

41. Tanaka Y, Matsuwaki T, Yamanouchi K, Nishihara M. Increased lysosomal biogenesis in activated microglia and exacerbated neuronal damage after traumatic brain injury in progranulin-deficient mice. Neuroscience. 2013;250:8-19.

42. Jian J, Tian QY, Hettinghouse A, Zhao S, Liu H, Wei J, Grunig G, Zhang W, Setchell KD, Sun Y, et al. Progranulin recruits HSP70 to betaGlucocerebrosidase and is therapeutic against Gaucher disease. EBioMedicine. 2016;13:212-24.

43. Schulze H, Sandhoff K. Sphingolipids and lysosomal pathologies. Biochim Biophys Acta. 2014;1841:799-810.

44. Shankaran SS, Capell A, Hruscha AT, Fellerer K, Neumann M, Schmid B, Haass C. Missense mutations in the progranulin gene linked to frontotemporal lobar degeneration with ubiquitin-immunoreactive inclusions reduce progranulin production and secretion. J Biol Chem. 2008;283:1744-53.

45. Wang J, Van Damme P, Cruchaga C, Gitcho MA, Vidal JM, Seijo-Martinez M, Wang L, Wu JY, Robberecht W, Goate A. Pathogenic cysteine mutations affect progranulin function and production of mature granulins. J Neurochem. 2010;112:1305-15.

46. Zhou X, Sun L, Bracko O, Choi JW, Jia Y, Nana AL, Brady OA, Hernandez JCC, Nishimura N, Seeley WW, Hu F. Impaired prosaposin lysosomal trafficking in frontotemporal lobar degeneration due to progranulin mutations. Nat Commun. 2017:8:15277.

47. Sardiello M, Palmieri M, di Ronza A, Medina DL, Valenza M, Gennarino VA, Di Malta C, Donaudy F, Embrione V, Polishchuk RS, et al. A gene network regulating lysosomal biogenesis and function. Science. 2009;325:473-7.

48. Palmieri M, Impey S, Kang H, di Ronza A, Pelz C, Sardiello M, Ballabio A. Characterization of the CLEAR network reveals an integrated control of cellular clearance pathways. Hum Mol Genet. 2011;20:3852-66. 
49. Rhinn $\mathrm{H}$, Abeliovich A. Differential aging analysis in human cerebral cortex identifies variants in TMEM106B and GRN that regulate aging phenotypes. Cell Syst. 2017:4:404-15.

50. Simons C, Dyment D, Bent SJ, Crawford J, D'Hooghe M, Kohlschutter A, Venkateswaran S, Helman G, Poll-The BT, Makowski CC, et al. A recurrent de novo mutation in TMEM106B causes hypomyelinating leukodystrophy. Brain. 2017;140:3105-11.

Ready to submit your research? Choose BMC and benefit from:

- fast, convenient online submission

- thorough peer review by experienced researchers in your field

- rapid publication on acceptance

- support for research data, including large and complex data types

- gold Open Access which fosters wider collaboration and increased citations

- maximum visibility for your research: over $100 \mathrm{M}$ website views per year 